\title{
Analysis and Warning Model of Logistics Risks of Cross-Border E-Commerce
}

\author{
Weizhe Mu iD \\ School of Management, Harbin University of Commerce, Harbin 150028, China \\ Correspondence should be addressed to Weizhe Mu; 102561@hrbcu.edu.cn
}

Received 17 November 2021; Revised 5 January 2022; Accepted 6 January 2022; Published 21 January 2022

Academic Editor: Daqing Gong

Copyright (c) 2022 Weizhe Mu. This is an open access article distributed under the Creative Commons Attribution License, which permits unrestricted use, distribution, and reproduction in any medium, provided the original work is properly cited.

Logistics is a key aspect of cross-border e-commerce (CBEC). If any link of logistics fails, the outcome of CBEC may fall shortage of expectation. Logistics risks are now major bottlenecks of CBEC development. Therefore, it is important to study CBEC logistics risks. This paper divides $\mathrm{CBEC}$ logistics risks into environmental risks, management risks, operation risks, and clearance risks and establishes a Bayesian model to warn CBEC logistics risks. Then, GeNIe software was employed to simulate the model, and an empirical analysis was conducted through forward prediction and reverse diagnosis. The results help enterprises to intuitively identify risk reducers, formulate effective risk control schemes, and thereby reduce CBEC logistics risks.

\section{Introduction}

In recent years, cross-border e-commerce (CBEC) has quickly developed into a popular trade form. In 2020, China's CBEC transaction volume reached 12.5 trillion yuan, accounting for $38.86 \%$ of the total import and export value. This volume is expected to reach 14.6 trillion yuan in 2021. Faced with the growing demand and logistics requirements of CBEC, the logistics industry is striving to open new channels, including international commercial express delivery, international special line logistics, and overseas warehousing. However, CBEC has a large coverage, involves various subjects, operates in a complex environment, and faces a huge policy impact, as compared with traditional e-commerce. It is very difficult for the $\mathrm{CBEC}$ to integrate the entire logistics process and realize unified management. As a result, the complex logistics process becomes more uncertain and poses a risky problem.

Logistics risks are a broad concept. In broad terms, logistics risks refer to the uncertainty of various logistics losses and reflect the outcomes and possible losses of various risks in logistics activities. In narrow terms, logistics risks refer to the deviation of expected outcome from the actual outcome. The greater the deviation, the larger the risks. CBEC cannot do without logistics, which, in return, affects the outcome of CBEC.

Domestic and foreign researchers have developed different approaches to warn logistics risks. For instance, Zhang and Ma [1] suggested that the various problems in CBEC can be solved efficiently by positioning overseas operations. Through social network analysis, Xue et al. [2] constructed a network model and a relationship matrix for multiple interactive risk factors and recognized the risk elements of CBEC ecosystem. Gao and Jia [3] analyzed the supply chain risks of 167 CBEC enterprises in Shanghai, Guangzhou, and Qingdao and identified the main risks of CBEC supply chain: online marketing, e-clearance, crossborder electronic payment, credit risks, and CBEC logistics risks. Sehoenherr et al. [4] adopted iterative analysis and structural analysis to identify logistics risks and evaluated the risk levels of the 17 identified risk factors through analytic hierarchy process (AHP). Taylor [5] constructed an operation risk evaluation system for logistics service providers, quantified the operation risks with the system, and presented risk control measures. Khan and Burnes [6] evaluated the operation risks of logistics and provided suggestions on logistics risk management. 
In the light of the reality, some experts and scholars have combined qualitative and quantitative methods to evaluate and warn CBEC logistics risks and established risk evaluation methods and warning systems. After analyzing the risk factors, Zheng et al. [7] built up a system of risk evaluation standards and carried out a case study on the CBEC operation modes of Chinese border exporters, using both qualitative and quantitative strategies. Zhang and Peng [8] explored the three risks of CBEC corporate management, namely, material flow, business flow, and capital flow. Based on activity quantification model, Giuffiida et al. [9] quantified the logistics costs of three CBEC logistics operation schemes and guided decision-makers to cut down logistics cost by preparing scientific CBEC logistics operation schemes. Zhang and Cao [10] held that the CBEC logistic mode contains lots of risks (political risks, market risks, information risks, and policy risks). To clarify the influence of each type of risks on CBEC logistics, they adopted fuzzy comprehensive evaluation (FCE) to compare the effects of different types of risks on CBEC logistics mode and evaluated and predicted the risks of CBEC logistics mode.

This paper identifies the indices for the warning of CBEC logistics risks, constructs a Bayesian model to warn such risks, and simulates the model on GeNIe software. In this way, the inducers of risk events were intuitively determined for CBEC logistics enterprises. Our research of CBEC logistics risks helps to identify the CBEC logistics risk events, promote the orderly development of the CBEC industry, find the risk factors of CBEC logistics, expand the research scope of risk management, formulate a government guidance mechanism for reducing CBEC logistics risks, provide policy makers with decision bases, cultivate a prevention system for CBEC logistics risks, and guide CBEC enterprises to evade risks and realize sustainable development.

\section{Indices for Warning of CBEC Logistics Risks}

Based on the features of CBEC logistics risks, this paper reviews the historical data of enterprises, evaluates the current status of CBEC industry, summarizes the experience of risk warning of the industry, and carries out questionnaire surveys, expert interviews, enterprise visits, and numerical modeling, thereby identifying various logistics risks faced by CBEC enterprises. The risk identification combines qualitative and quantitative analyses. From the identified risks, four kinds of risks were selected for risk warning analysis, including environmental risks, management risks, operation risks, and clearance risks (Figure 1). The environmental risks come from the external environment of the CBEC logistics. The management risks stem from CBEC logistics management. Operation risks are associated with the operational links of logistics. Clearance risks are related to the import/ export customs clearance of CBEC goods.

2.1. Environmental Risks. Environmental risks stem from one of the unavoidable external factors of CBEC logistics operation. The environment can be divided into natural environment and social-cultural environment. The former is the basis of the latter, while the latter is derived from the former. Because CBEC is highly networked, informatized, and cross-regional, the logistics operation will be constrained by different environments to varied degrees, including natural environment, legal environment, and economic environment. These factors directly or indirectly influence CBEC logistics operation and may even lead to adverse effects. These risks can be quantified with prior probabilities. The quantification process will be detailed in subsequent sections.

2.1.1. Natural Risks. Relative to social environment, the natural environment refers to the environment formed by natural things, such as soil, region, and climate. Natural risks exert an objective impact on logistics. Take land transport as an example. The rolling friction of truck wheels varies with highway conditions. The cargo hoisting of a crane is affected by weather and wind power. The freight volume must meet the provisions of highway policies and fall within the bearing capacity of highway surface and bridges. During air transport, the wind direction and air flow must be given special attention. Due to the high value of air cargoes, air transport is relatively costly and sensitive to environmental risks. As for marine transport, the vessels might suffer from accidents like capsizing and stranding.

2.1.2. Legal Risk. The legal environment refers the various laws, regulations, and regulations issued by the national or local government. To a certain extent, the legal environment can regulate the operation of CBEC enterprises. Because $\mathrm{CBEC}$ operates across countries and regions, its operation is inevitably affected by the different legal systems of different countries. Before CBEC logistics, the relevant enterprises need to get familiarized with the legal environment of the other countries. In this way, the enterprises can operate strictly in accordance with the law and predict the market demand and trend based on the changes in legal provisions. Otherwise, CBEC enterprises will easily commit illegal or default behaviors during cross-border trade.

2.1.3. Economic Risks. The economic environment refers to the external socioeconomic conditions for enterprise development, including consumer income level, consumer spending model, consumption structure, consumer savings and credit cards, level of economic development, economic development in specific fields/industries, and urbanization level. Any form of trade is affected by its economic environment. CBEC logistics is no exception. Because CBEC logistics operate across countries and regions, it is influenced by both international and domestic economic conditions. The international economic environment is related to CBEC logistics and national economic development. The changes in financial environment and industrial structure affect the workload of CBEC enterprises, bringing them potential risks. For example, any change of exchange rate, the volatility of the stock market, and global/local financial crisis add difficulty or burden to corporate financing. The domestic 


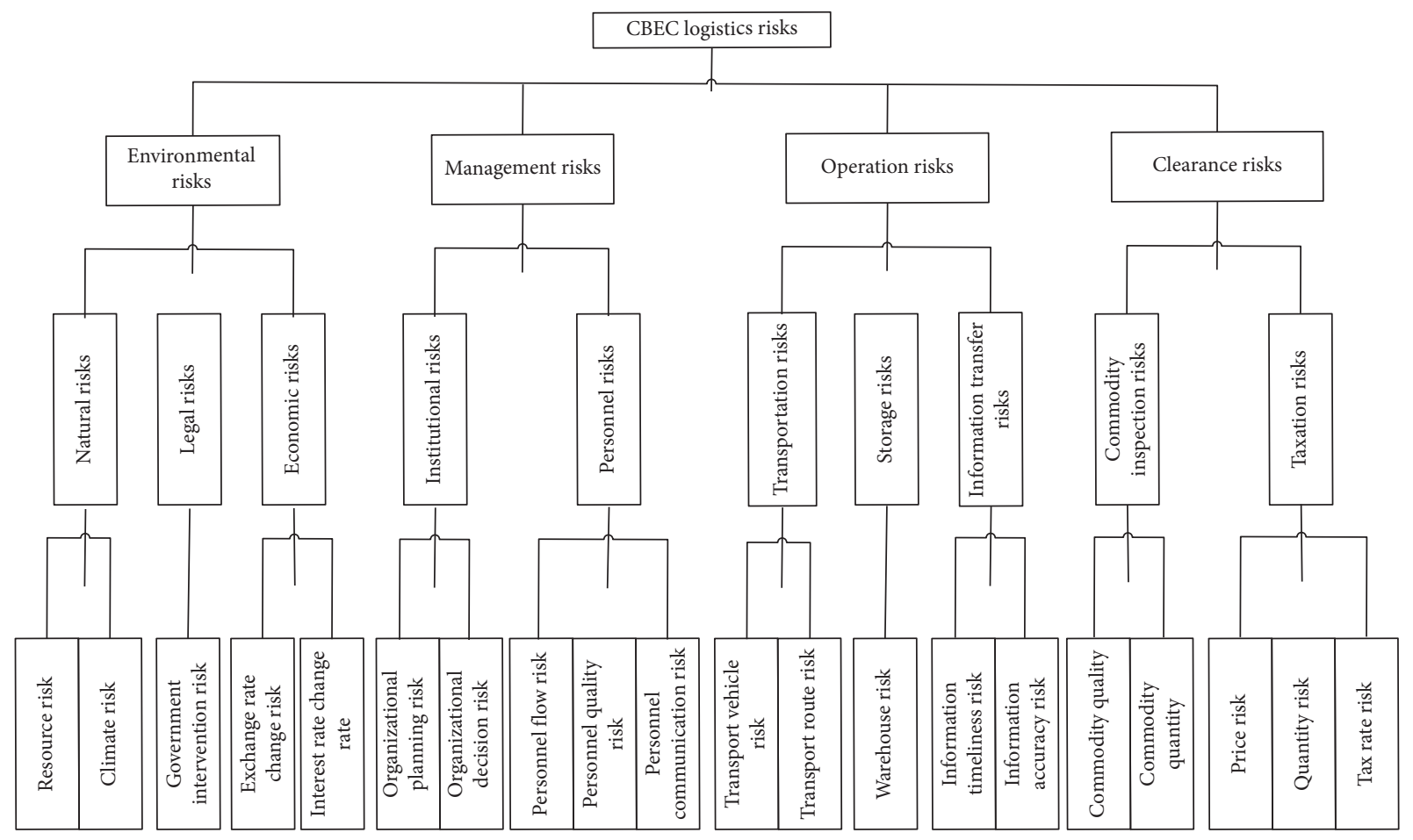

FIGURE 1: Risk warning indices.

economic environment stands for the national economic indices related to the current financial system of the host country. CBEC logistics develops faster if the economy is prosperous and slower if the economy enters a recession.

2.2. Management Risks. Management risks mean the management level is affected by information asymmetry, improper management, and incorrect judgment during the management process. These risks exist in each detail of the management system. They can be divided into four parts: cultural quality of managers, organizational structure, corporate culture, and management flow. Here, management risks are merely analyzed from institutional risks and personnel risks.

2.2.1. Institutional Risks. The institution of an enterprise is the organic whole of all the components that make the enterprise an independent organization. It is the cornerstone of corporate development and the guideline for corporate operation and working. If a CBEC enterprise has a chaotic institution, its logistics operation will be at stake.

2.2.2. Personnel Risks. Personnel is an essential factor in any risk analysis. CBEC logistics is greatly affected by personnel, because it involves various personnel, such as traders, custodians, logistics personnel, regulatory personnel, and customer service staff.

2.3. Operation Risks. Operation risks refer to the various risks that may present in the specific operation of CBEC logistics. These risks are related to the operational links of logistics.
2.3.1. Transportation Risks. During transportation, many unmeasurable risks might be induced by external and internal factors, such as traffic accidents, and poor road conditions. There are also risks during the transportation. For example, cross-border logistics usually transports goods by air. But the goods are rarely delivered directly to the destination by one transport means alone. In many cases, several transport means must be combined to complete the delivery. Many problems may arise in the transit process, such as the loss of goods, the damage of goods (caused by violent sorting), and delayed delivery.

2.3.2. Storage Risk. In terms of storage, the risk occurs either in stock or enroute. In-stock storage risk mainly refers to the various storage risks in the warehouse: operation mistake, loss of stocked goods, pest attack, draught, and flood. Enroute storage risk stands for the damage of goods during the delivery process.

2.3.3. Information Transfer Risks. During the transfer of logistics information, information asymmetry or distortion easily occurs, especially when the central nodes forward information and receive feedbacks. Both information asymmetry and information distortion push up the risk cost.

2.4. Clearance Risks. Clearance, i.e., customs clearance, means import, export, and transit goods, must be declared to the customs after reaching a country/region. The importer/exporter/forwarder should go through the various procedures specified by the customs, and perform every duty required by the law. The goods will not be released 
before all the duties have been fulfilled, and all the formalities (declarable, inspection, taxation, etc.) have been gone through. Similarly, the transport tools of import/ export/transit goods should also be declared to the customs before leaving/entering the border. The importer/exporter/ forwarder needs to handle the relevant formalities and win the approval of the customs. During the clearance, the goods must be imported/exported/transferred under the supervision of the customs, rather than circulating freely. This paper analyzes clearance risks in two dimensions: commodity inspection risks and taxation risks.

2.4.1. Commodity Inspection Risks. The import and export commodity inspection is an independent inspection agency set up by the country or redigested at the government. The agency is responsible for inspecting and approving the quality, standard, health state, and quantity of import/export goods and issue proofs. The purpose is to provide a thirdparty certification that ensures the legitimate rights and interests of the parties in foreign trade. During cross-border trade, any goods must go through the inspection and quarantine before entering or leaving the border of the relevant countries. Two problems often occur during the commodity inspection. First, the customs screen the goods carefully to check if there are any fake goods. Fake goods are strictly prohibited by China's customs policies. Second, many goods are not allowed to be exported, such as batteries and precious metals.

2.4.2. Taxation Risks. Taxation risks mainly refer to the taxation losses of goods caused by the changes of policies and interest rate in relevant countries. Meanwhile, some traders would purposefully evade their taxation duty. To prevent the violation of laws and regulations, CBEC enterprises must fully understand the tax policies of all countries and carefully inspect the goods of their clients.

\section{Model Construction}

3.1. Node Selection. Our Bayesian model contains three kinds of nodes: risk inducers (parent nodes) and risk indices (child nodes). The parent nodes were obtained through observation or statistical analysis. They directly represent the occurrence of a risk event. The probability of child nodes was calculated by Bayesian rules. From the above analysis, it could be inferred that there are four types of CBEC logistics risks environmental risks, management risks, operation risks, and clearance risks. Specifically, environmental risks include natural environment, legal environment, and economic environment; management risks involve institution dimension and personnel dimension; operation risks cover transportation process risk, storage process risk, and information transfer risks; clearance risks encompass commodity inspection risks and taxation risks. On this basis, a Bayesian model for risk warning was created as shown in Figure 2.
3.2. Assignment of Prior Probabilities. After obtaining the Bayesian model for the warning of CBEC logistics risks, the authors clarified the relationship between different factors. Then, it is necessary to determine the prior probability of each risk factor. The prior probability is a probability obtained by analyzing the previous experience. That is, prior probability relies on subjective empirical estimation and needs the deduction based on the existing knowledge. In this paper, the prior probabilities are calculated, according to the data in the journals, financial magazines, and financial newspapers reporting CBEC logistics risk events in 2009-2019, and referring to the experience of relevant practitioners and logistics experts. During the actual use, CBEC enterprises can further correct the prior probabilities according to their actual data.

During node setting, different risk levels were configured, according to the unique features of each type of nodes. For example, clearance risks can be classified into five states: strong, relatively strong, medium, relatively weak, and weak. A strong clearance risk means the customs clearance faces a serious risk; a relatively strong clearance risk means the customs clearance faces a certain level of risk; a medium clearance risk means the customs clearance faces a general risk; a relatively weak clearance risk means the customs clearance faces a risk with a limited impact; a weak clearance risk means the customs clearance faces a negligible risk. The origin node of clearance risks, namely, price risk, could be divided into two kinds: occurrence and nonoccurrence; for a transitional node (e.g., taxation risks) between the two, there are three possible risk states: strong, medium, and weak. Then, each node was assigned a value. As mentioned in the previous section, the node values were determined based on the literature, experience, and the results of questionnaire survey (Tables 1-3).

\section{Warning Intervals and Warning Lights}

Lamp signal method provides a simple yet effective way to predict logistics risks. By this method, the level of logistic risks is indicated by lights of different colors. The logistics risk warning by this method can be implemented in the following steps:

(1) Determine the detection indices of the warning mechanism.

This paper performs warning monitoring of all intermediate and destination nodes.

(2) Set the light color for each node state.

From high to low, the risk levels were indicated by red, orange, yellow, green, and blue lights, in turn.

(3) Compute the critical level of each node.

The critical value refers to the boundary value of the risk from one level to the next superior level. The boundary value is often determined by empirical method. This is a key step of lamp signal simulation.

Let $a, b$, and $c$ be the high-, medium-, and low-risk probabilities of an intermediate node, respectively. The 


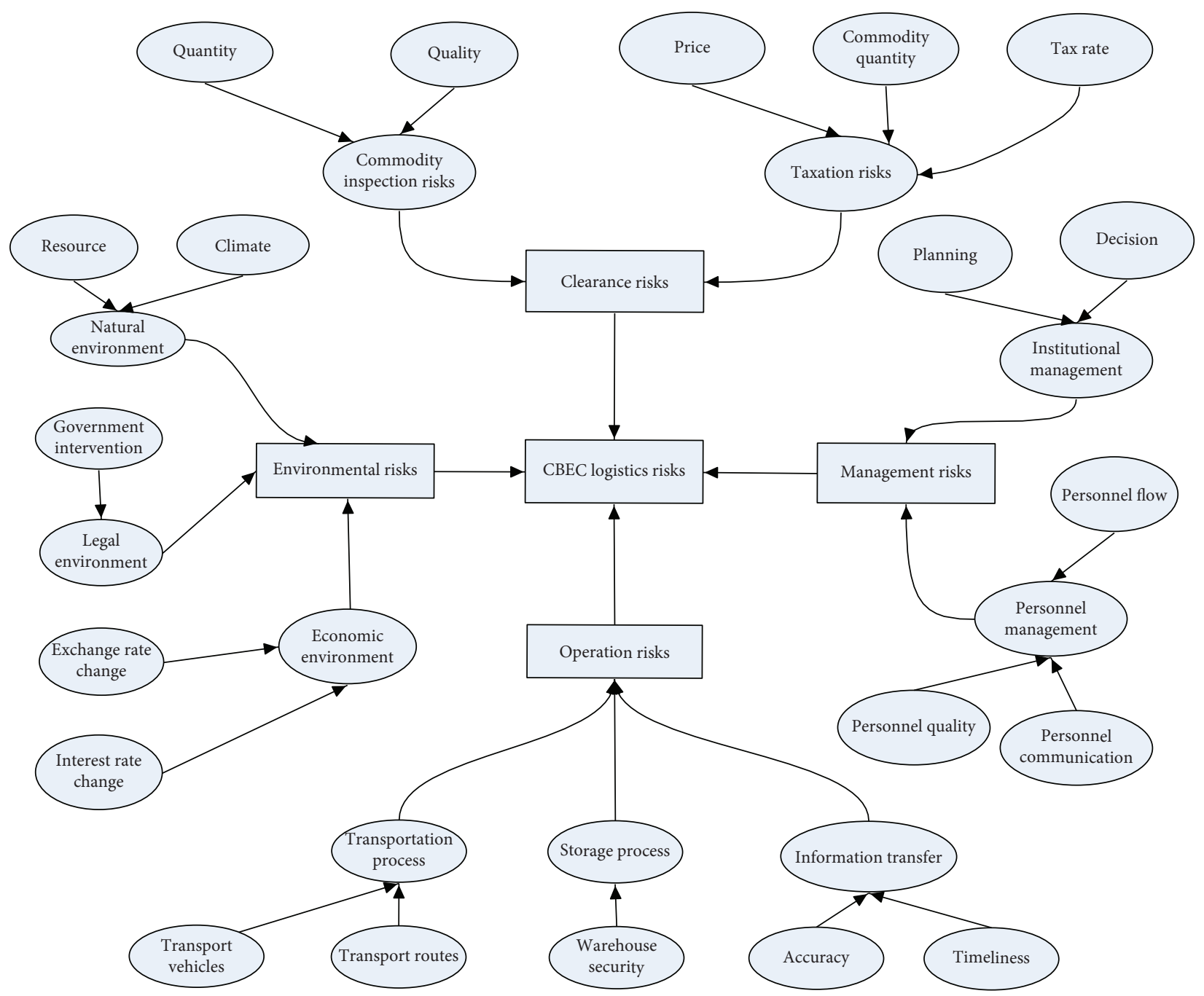

FIGURE 2: Bayesian model for the warning of CBEC logistics risks.

TABLe 1: Value assignment to origin nodes.

Name of the node

Prior probability (\%) Occurrence

Nonoccurrence

Resource shortage

Climate change

Government intervention

Exchange rate change

Interest rate change

Organizational planning

Organizational decision

Personnel flow

Personnel quality

Personnel communication

Transport vehicles

Transport routes

Warehouse problem

Information timeliness

1

1

1

5

2

2

2
3

3

2

Information accuracy

Export commodity quantity

Commodity quality

Commodity price

Commodity quantity 
TABLE 2: Value assignment to destination nodes.

\begin{tabular}{lccccc}
\hline Name of the node & Strong & Relatively strong & $\begin{array}{c}\text { Prior probability (\%) } \\
\text { Medium }\end{array}$ & Relatively weak \\
\hline Environmental risks & 9 & 11 & 28 & 27 \\
Management risks & 16 & 22 & 24 & 20 & 25 \\
Operation risks & 12 & 13 & 29 & 24 & 19 \\
Clearance risks & 17 & 23 & 24 & 18 \\
CBEC logistics risks & 10 & 13 & 34 & 25 \\
\hline
\end{tabular}

TABLE 3: Value assignment to intermediate nodes.

\begin{tabular}{lccc}
\hline \multirow{2}{*}{ Name of the node } & \multicolumn{3}{c}{ Prior probability (\%) } \\
& High & Medium & Low \\
\hline Natural risks & 21 & 20 & 59 \\
Legal risk & 14 & 20 & 67 \\
Economic risks & 11 & 20 & 56 \\
Institutional risks & 22 & 21 & 58 \\
Personnel risks & 12 & 41 & 47 \\
Transportation risks & 23 & 21 & 56 \\
Storage risk & 16 & 19 & 65 \\
Information transfer risks & 30 & 23 & 47 \\
Commodity inspection risks & 25 & 23 & 51 \\
Taxation risks & 16 & 42 & 42 \\
\hline
\end{tabular}

values of $a, b$, and $c$ were empirically assigned as $0.5,0.3$, and 0.2 . Hence, $A=0.5 \times a+0.3 \times b+0.2 \times c$. The theoretical range of $A$ can be derived as $[20,50]$.

Let $a, b, c, d$, and $e$ be the high-, relatively high-, medium, relatively low-, and low-risk probabilities of a destination node. The values of $a-e$ were empirically assigned as $0.4,0.3$, $0.15,0.1$, and 0.05 . Hence, $B=0.4 \times a+0.3 \times b+0.15 \times c$ $+0.1 \times d+0.05 \times e$. The theoretical range of $\mathrm{B}$ can be derived as $[5,40]$. Table 4 shows the warning intervals.

\section{Model Simulation}

Through GeNIe simulation, the prior probabilities of our model can be obtained (Figure 3).

As shown in Figure 3, after the model parameters were given values based on the survey data, the warning value of the logistics risk level for CBEC enterprises was 16.45, belonging to Level II. This means the enterprise has a few risks. Specifically, the warning value of environmental risks was 14.95, belonging to Level I. Hence, environmental risks are normal. The warning value of operation risks was 16.5 , belonging to Level II. Thus, there are some operation risks in the enterprises. The warning value of management risks was 19.55, belonging to Level III. The warning value of taxation risks was 19.95, also belonging to Level III. Therefore, many CBEC enterprises face management risks and taxation risks. Measures should be taken to guard against these two types of risks.

\section{Empirical Analysis}

The Bayesian-based warning mechanism for CBEC logistics risks evaluates the risk level of an enterprise via two paths: forward prediction and reverse diagnosis. Forward prediction is to derive the state of the child nodes from the changes of the parent node and judge the degree of impact of different parent nodes on child nodes, revealing the influence of different risk factors over CBEC logistics risks. Through Bayesian inference, reverse diagnosis derives the parent node state from the changes in child nodes and reversely deduces the inducements of various risk events. On this basis, suitable risk treatment measures can be adopted timely to prevent secondary occurrence and lower risk losses. Different measures should be taken to address the varied causes of risk events and evaluated against different risk events. This process will be discussed during the empirical analysis.

6.1. Forward Prediction. According to the varied levels of actual risk factors, forward prediction adjusts the values of the key risk inducers in Bayesian model and transmits the changes to the other nodes through forward reasoning. Then, the changes of each node are observed, and the influence of a changing node on the other node is evaluated. In essence, forward prediction is a forecast of risk events. Through forward prediction, risk managers can learn the risk levels of corporate logistics in advance and take countermeasures as early as possible.

Take CBEC enterprise $A$ as an example. During a transaction, the enterprise failed to deliver goods, due to a delay in the transmission of order information. As a result, the enterprise suffered a huge loss from inventory backlog. Then, the probability of information timeliness rose from $15 \%$ to $100 \%$, and the probability of warehouse problem induced by inventory backlog increased from $8 \%$ to $100 \%$ (Figures 4 and 5).

Before the risk occurred, the warning value of CBEC logistics risks as 16.45, belonging to Level II (normal level); after the occurrence of the operation risk, the warning value increased to 17.45 , belonging to Level III.

The enterprise investigated and affixed the responsibility for the delay in the transmission of order information. Through the investigation, the delay was attributed to a decision mistake of senior managers and the dereliction of duty by logistics personnel. Therefore, the responsible personnel were dismissed. In this case, the probability of organizational decision risk increased from $3 \%$ to $100 \%$ and that of personnel flow risk rose from $2 \%$ to $100 \%$. The warning value of CBEC logistics risks did not change significantly (from 16.45 to 16.9 ), suggesting that the risk level remains unchanged (Figures 6 and 7). 
TABLE 4: Warning intervals.

\begin{tabular}{|c|c|c|c|c|}
\hline $\begin{array}{l}\text { Risk } \\
\text { level }\end{array}$ & $\begin{array}{l}\text { Critical value for intermediate } \\
\text { nodes }\end{array}$ & $\begin{array}{l}\text { Critical value for destination } \\
\text { nodes }\end{array}$ & Light & Control measures \\
\hline $\bar{I}$ & {$[20,25.5)$} & {$[5,15)$} & Blue & The risk belongs to the normal range \\
\hline II & {$[25.5,27.5)$} & {$[15,17)$} & Green & Safety warning should be given \\
\hline III & {$[27.5,28.5)$} & {$[17,23)$} & Yellow & $\begin{array}{c}\text { Corresponding measures should be taken to solve the } \\
\text { hazardous risk }\end{array}$ \\
\hline IV & {$[28.5,30.5)$} & {$[23,30)$} & Orange & $\begin{array}{c}\text { Corresponding measures should be taken to solve the } \\
\text { unwanted risk }\end{array}$ \\
\hline $\mathrm{V}$ & {$[30.5,50)$} & {$[30,40)$} & Red & Immediate measures should be taken \\
\hline
\end{tabular}

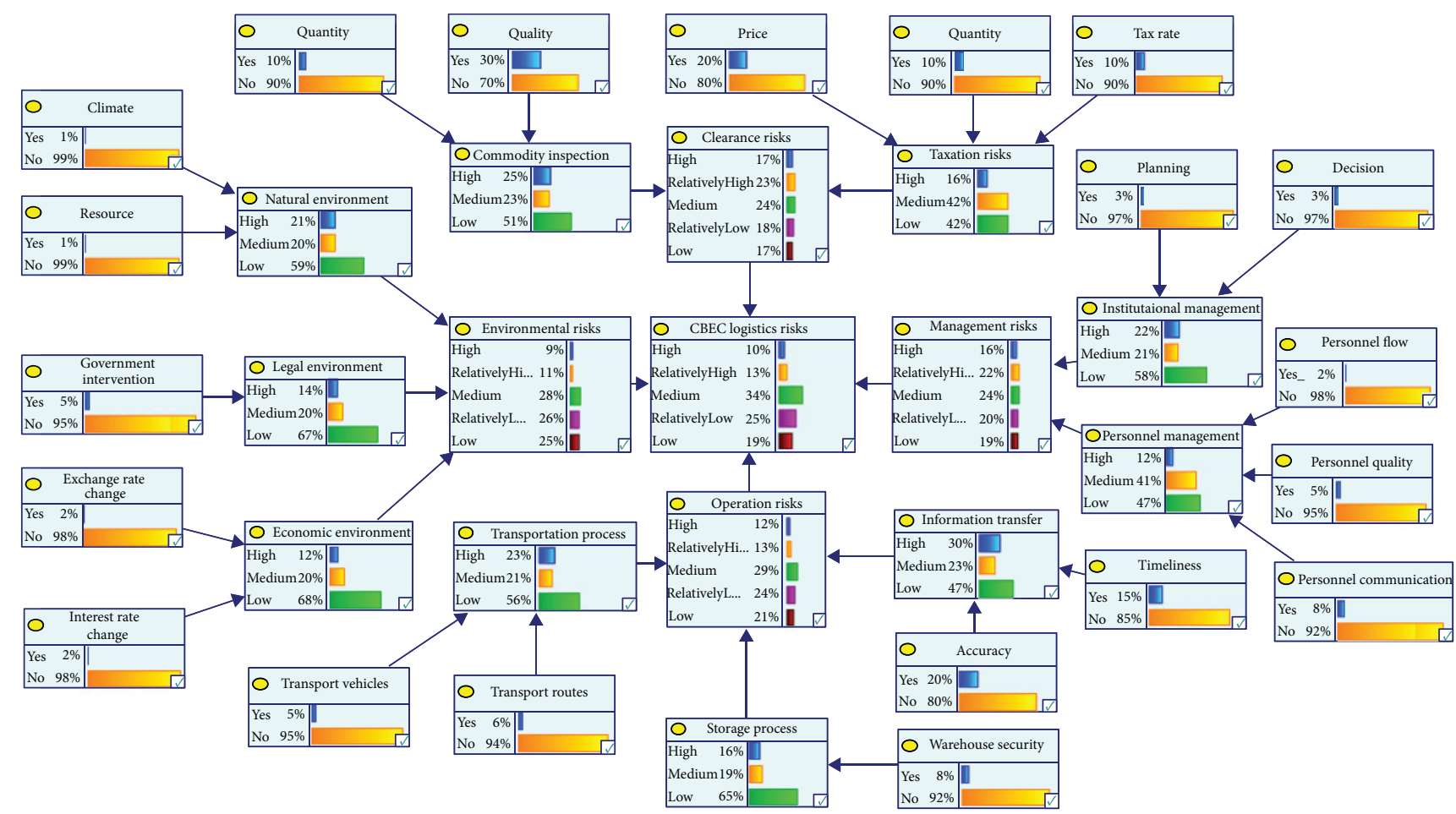

FIgURE 3: Prior probabilities of our warning mechanism for CBEC logistics risks.

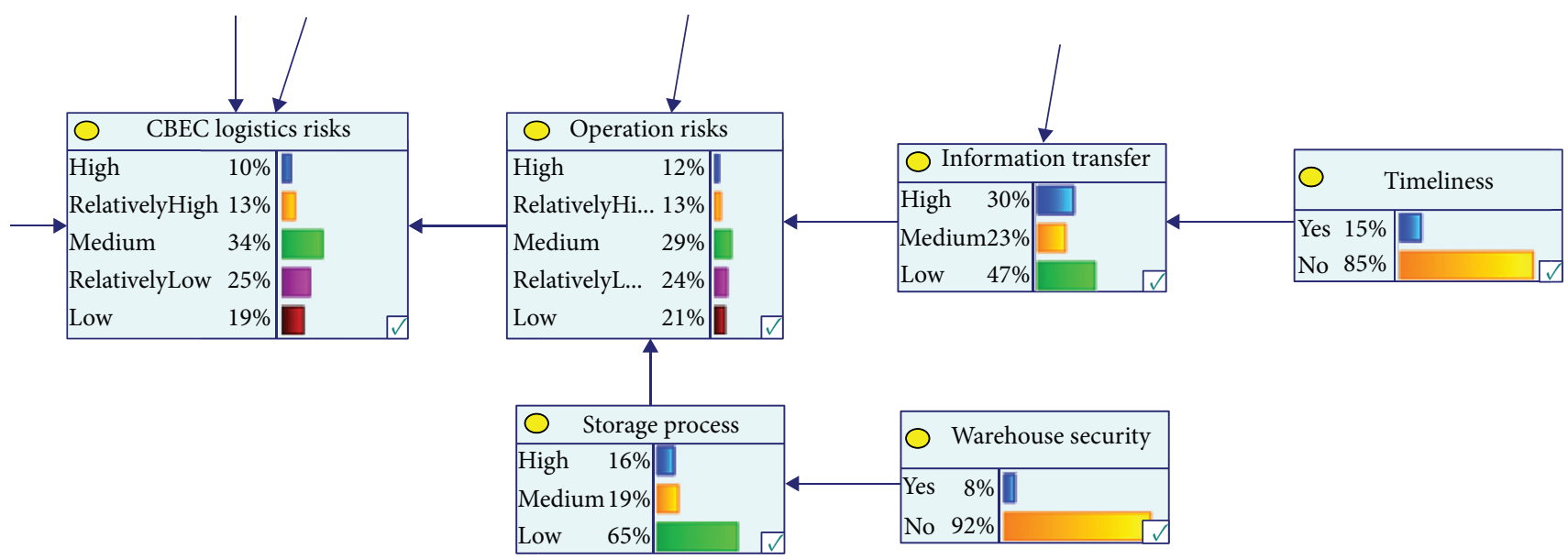

FIGURE 4: Before the operation risk. 


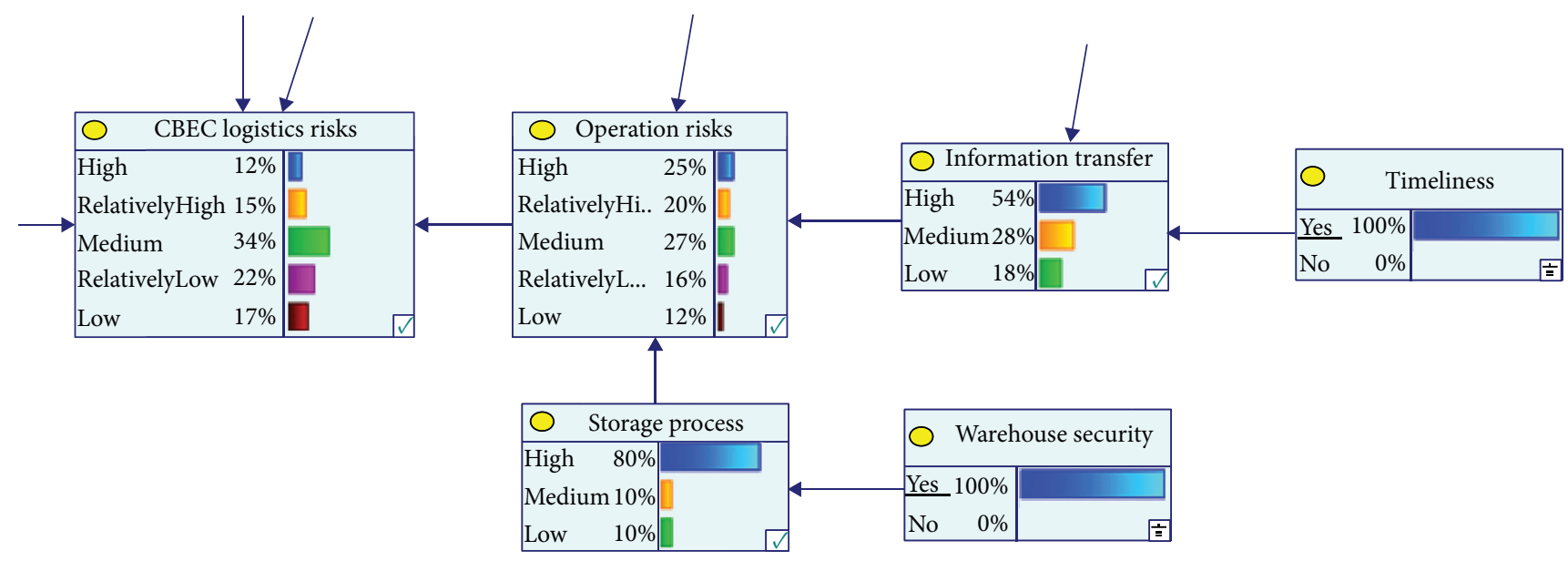

FIGURE 5: After the operation risk.

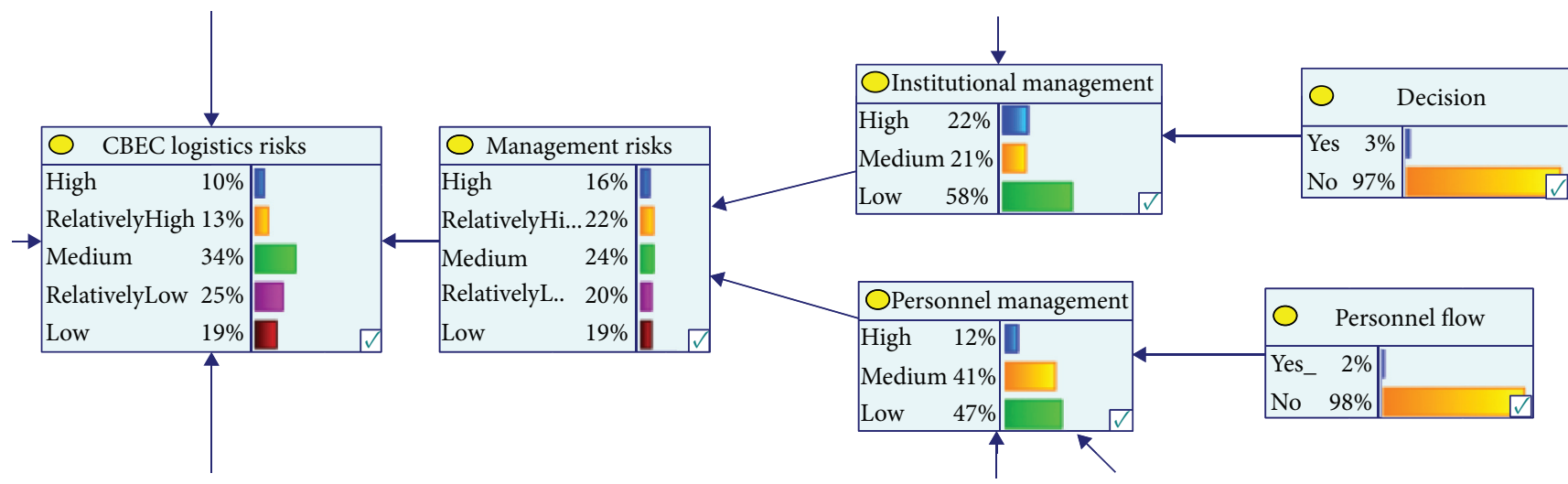

Figure 6: Before dismissal.

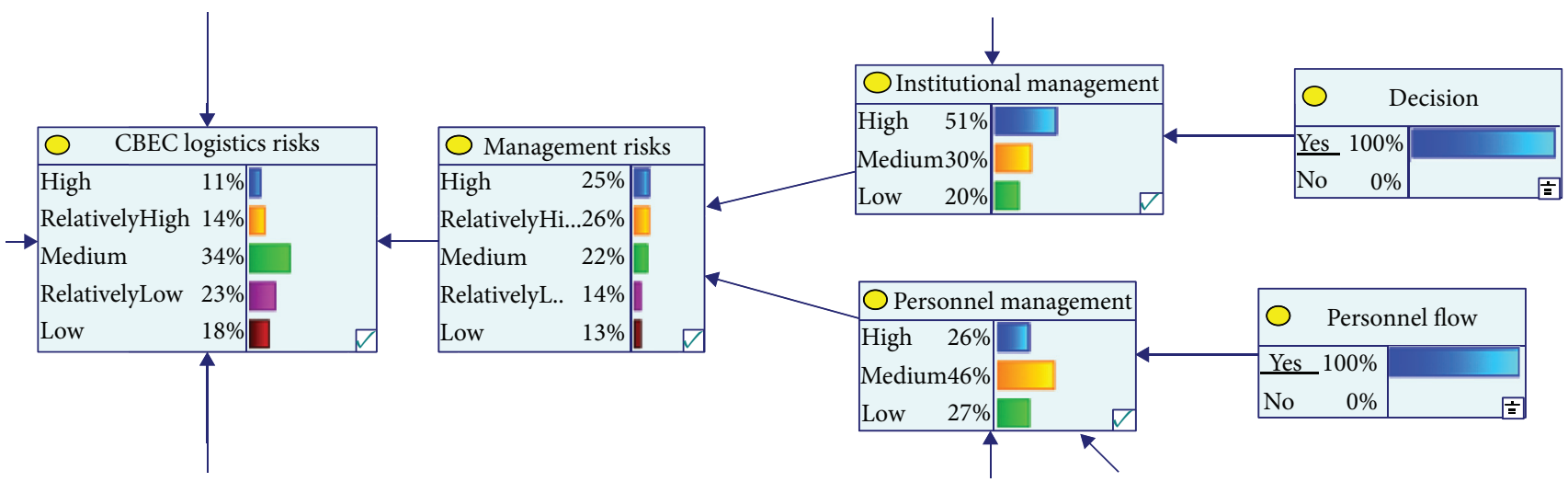

Figure 7: After dismissal.

The two changes of risk levels indicate that, for the same risk event, operation risks have deeper influence over CBEC logistics risks than management risks. Therefore, enterprise A should focus more on operation risks, as it prepares risk prevention measures.

6.2. Reverse Diagnosis. Reverse diagnosis first changes the values of key risk indices, then observes the situation of the other nodes, and derives the level of the other risk factors. It can also be defined as follows: when the outcome of the risks is known, the identification of inducers and their posterior probabilities is through Bayesian diagnosis.

When the probabilities of logistics risks of CBEC enterprise A changed from $10 \%, 13 \%, 34 \%, 25 \%$, and $19 \%$ to $30 \%, 25 \%, 31 \%, 8 \%$, and $6 \%$, the risk probabilities of the four nodes changed accordingly (Figures 8 and 9). Among them, the warning values of clearance risks and management risks 


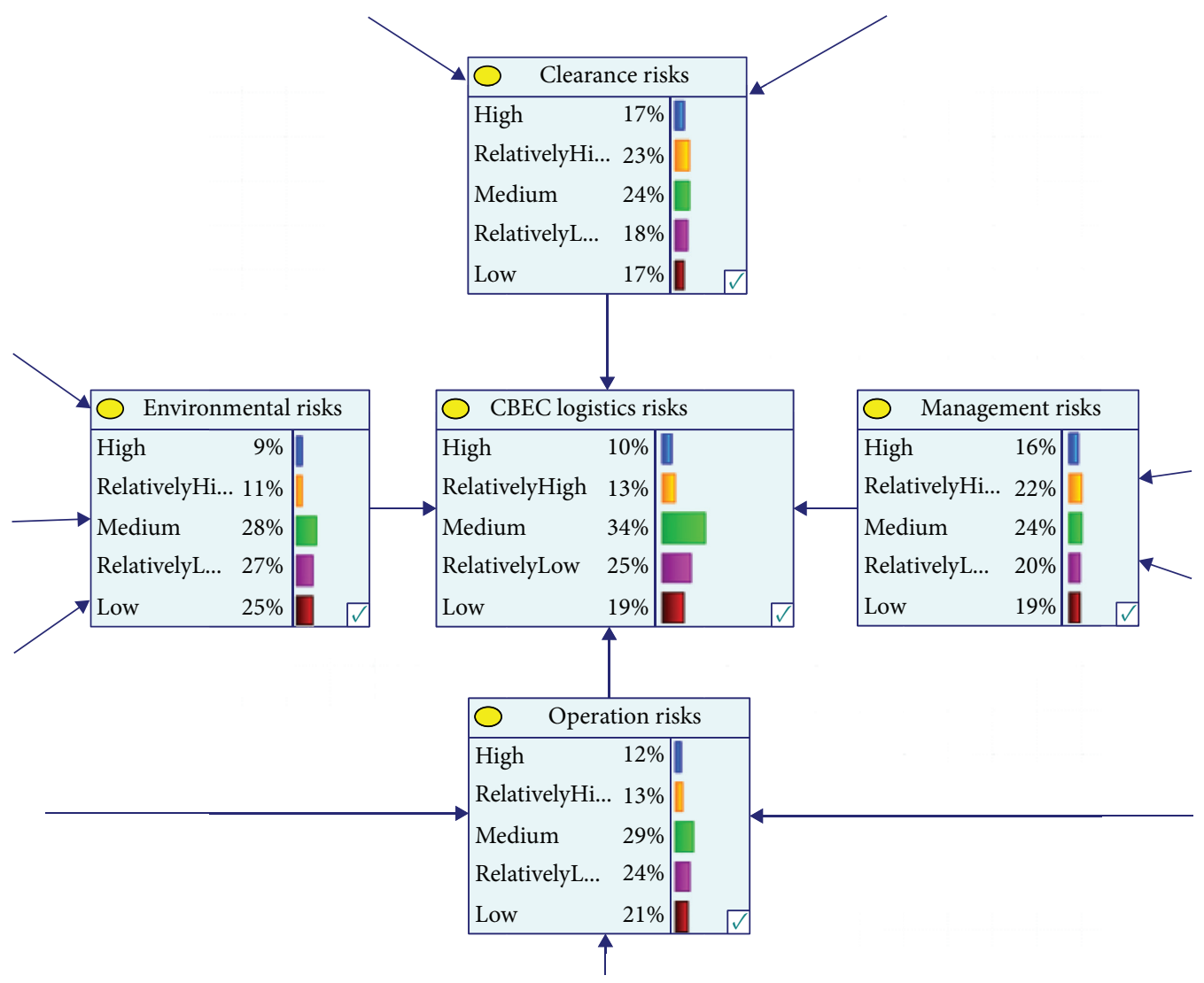

FIGURE 8: Before logistics risks.

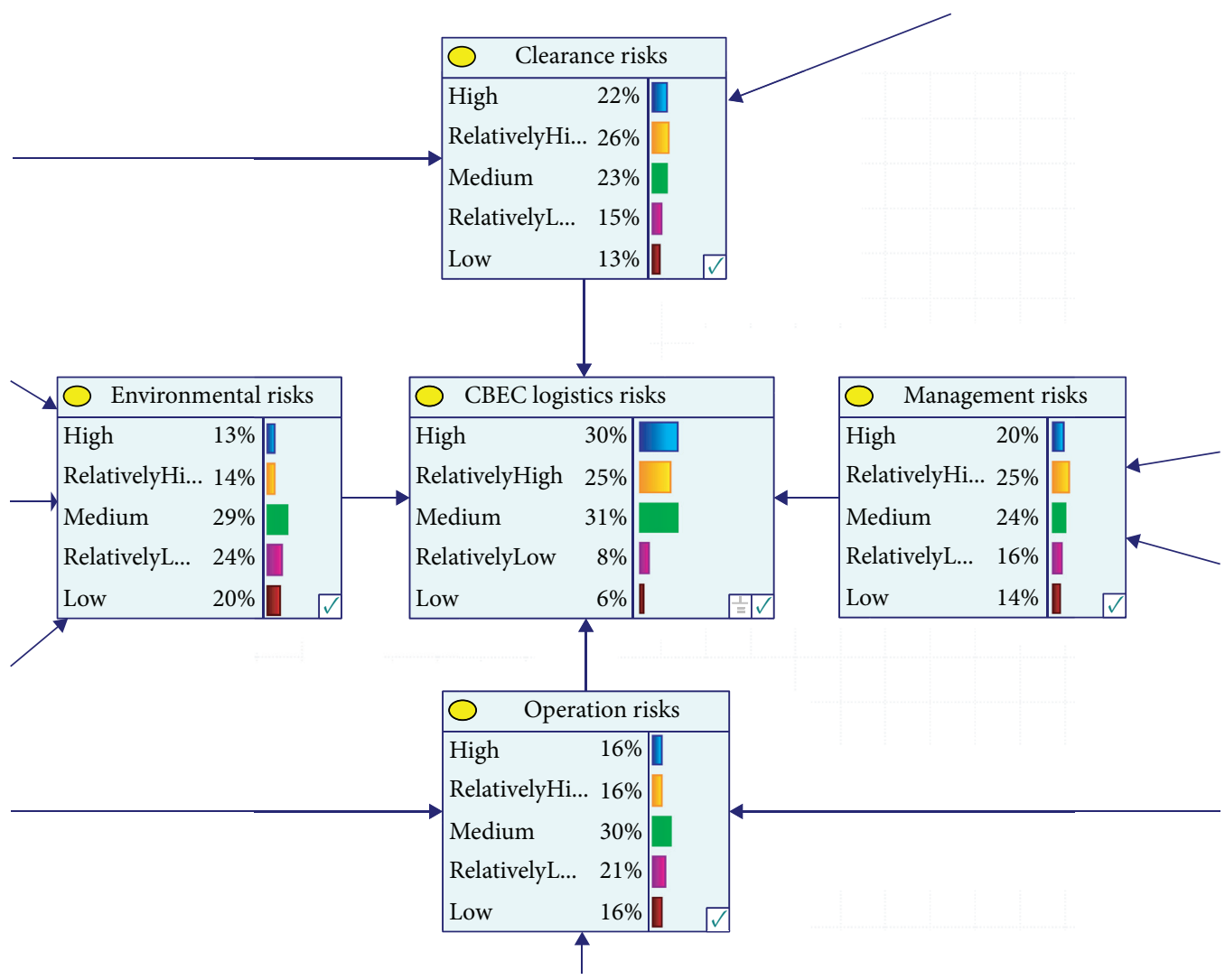

Figure 9: After logistics risks. 
varied relatively slightly; i.e., the risk levels did not change much. By contrast, the warning value of environmental risks changed more significantly than any other type of risks, jumping from Level I to Level III. The warning value of operation risks changed slightly from Level II to Level III. Overall, the change in CBEC logistics risks is primarily driven by environmental factors, followed by the maloperations in logistics. The enterprise should recognize the environmental impact of the transaction and pinpoint the loopholes of logistics operation. On this basis, remedial measures and treatment schemes should be prepared to prevent the same risk from occurring again.

\section{Conclusions}

The rapid development of CBEC has intensified the competition among enterprises and highlighted logistics risks. Therefore, it is critical to accurately warn logistics risks. After a thorough literature review and repeated visits to typical CBEC enterprises in China, this paper chooses to analyze risk warning from such four aspects as environmental risks, management risks, operation risks, and clearance risks. A total of 35 indices were designed for the four aspects and organized into a Bayesian model for the warning of CBEC logistics risks. Five light colors were designed for different warning intervals of CBEC logistics risks. Each color corresponds to a risk level. From high to low, the five colors indicate that immediate measures should be taken, corresponding measures should be taken to solve the unwanted risk, corresponding measures should be taken to solve the hazardous risk, safety warning should be given, and the risk belongs to the normal range, respectively. Next, GeNIe software was adopted to simulate our model. After that, an empirical analysis was carried out through forward prediction and reverse diagnosis. The results show that, for the same risk event, operation risks have deeper influence over CBEC logistics risks than management risks. Taking enterprise $\mathrm{A}$ as an example, its CBEC logistics risks are influenced deeper by operation risks than management risks. To reduce risk losses, enterprise A should focus more on operation risks, as it prepares risk prevention measures. The above theoretical and empirical evidence show that the proposed risk warning model helps enterprises to intuitively identify risk inducers and prevent the risks in time. During the actual use of our model, the enterprises must adjust the parameter values according to their own situation, in order to derive their own risk levels, discover risk problems, and make pertinent treatment measures, thereby reducing the risk losses.

\section{Data Availability}

The data used to support the findings of this study are available from the corresponding author upon request.

\section{Conflicts of Interest}

The author declares that there are no conflicts of interest.

\section{Acknowledgments}

This work was funded by 2021 Philosophy and Social Science Thinktank Project, Heilongjiang Province, China (Grant number: 21ZK021).

\section{References}

[1] X. Zhang and T. Ma, "Difficulties and countermeasures about China's cross-border e-commerce logistics," JI Economic Management, vol. 37, no. 5, pp. 51-54, 2015.

[2] C. G. Xue, M. N. Yan, and D. Zhang, "Risk factors identification of cross-border e-commerce ecosystem based on social network analysis," Finance and Accounting Monthly, vol. 16, pp. 113-119, 2019.

[3] X. Gao and L. T. Jia, "Research on the supply chain risk of the enterprises of cross-border electronic commerce in the structural equation Modeling,Based on 167 enterprises located in Shanghai, Guangzhou,Qingdao and other cities," Shanghai Economic Review, vol. 5, pp. 76-83, 2016.

[4] T. Schoenherr, V. M. Rao Tummala, and T. P. Harrison, "Assessing supply chain risks with the analytic hierarchy process: p," Journal of Purchasing and Supply Management, vol. 14, no. 2, pp. 100-111, 2008.

[5] R. W. Taylor, "Logistics risk in the stryker brigade combat team," Army Logistician, vol. 1, 2004.

[6] O. Khan and B. Burnes, "Risk and supply chain management: creating a research agenda," International Journal of Logistics Management, pp. 302-321, 2007.

[7] X. X. Zheng, D. F. Li, Y. Wang, and J. C. Liu, "Logistics Risk Assessment of China's export cross-border e-commerce," Business and Economic Research, vol. 23, pp. 68-69, 2016.

[8] Y. T. Zhang and Y. Peng, "Research on operation risk early warning positioning of cross-border e-commerce enterprises," Finance and Accounting Monthly, vol. 24, pp. 72-75, 2016.

[9] M. Giuffrida, R. Mangiaracina, A. Perego, and A. Tumino, "Logistics solutions to support cross border E-commerce towards China: the case of the apparel industry," Business Models and ICT Technologies for the Fashion Supply Chain, pp. 163-177, 2017.

[10] D. Zhang and W. J. Cao, "Model classification and risk assessment of cross-border E-commerce logistics in China's foreign trade," Journal of Henan Normal University (Philosophy and Social Sciences Edition, Bimonthly), vol. 46, no. 5, pp. 53-59, 2019. 